\title{
Barriers To Uptake of Childhood Routine Immunization In Nyeri County, Kenya
}

\author{
Njeru S. K ${ }^{1}$, Kagoiyo S. W ${ }^{2}$, Dennis Butto ${ }^{1}$ \\ ${ }^{1}$ (Department Of Clinical Medicine, Kirinyaga University, Kenya) \\ ${ }^{2}$ (Kerugoya County Refferal Hospital, County Government of Kirinyaga, Kenya)
}

\begin{abstract}
Routine immunization coverage in the developing world is as low as 60\% compared to the developed world where it way above $80 \%$. This has caused deaths among children and even left others with permanent disability. This A descriptive cross sectional study was conducted in Mukurweni and Tetu Sub counties, Nyeri County, Kenya to determine factors influencing routine immunization coverage among children. The study revealed that there was a moderate coverage in Mukurweini and high coverage in Tetu Division. The higher number of immunized children in both divisions was in the age bracket of $1-4$ months (88.7\%) and reducing to $0.05 \%$ during 12 months of children's age. This indicates that fewer children appeared for immunizations as they advance in age. The reasons cited by mothers /caretaker for low uptake of immunization included: fear of side effects associated with vaccines such as fever, fear that immunization would cause impotence in their children, religious beliefs and lack of money to go to health facilities. Negative attitude of some health workers was cited in both divisions as an operational factor impeding routine immunization. From the above results, it can be said most of these barriers are socio cultural rather than health system obstacles. Community education and advocacy aimed at creating awareness and the vital role of childhood immunization would be key to improving coverage.
\end{abstract}

Keywords: Immunization, Coverage, Immunizable diseases, Polio

\section{Introduction}

Routine immunization coverage among children in developing countries is below the WHO and UNICEF target of $80 \%$ which leads to many children suffering from infectious parasitic and immunizable diseases which are by far the leading causes of morbidity and mortality in the developing countries [1]. There are many variations reported in developing countries in infant mortality and life expectancy due to lack of immunization [2,3]. It has been globally estimated that globally 5 million children die due to lack of immunization of vaccine preventable diseases [4]. Many other children are disabled by complications of immunizable diseases, thus raising child morbidity.

The immunizable diseases among children are tuberculosis, poliomyelitis, diphtheria, pertussis, tetanus, viral hepatitis, haemophilus influenza and measles. These diseases are divided into 3 categories for their effective management. There are those diseases targeted for elimination, others for eradication and those that are for control [5]. The vaccines are preparations which contain antigens that when administered for specific diseases provide a lifelong immunity by triggering an immune reaction[6]. In Kenya, the expanded program on immunization (EPI) launched in June 1980 helped to raise the immunization coverage form $30 \%$ of initiation to $80 \%$ by $1993[7])$.

Eastern blocks of the world, where Kenya and other East African countries belong have adopted the Measles supplemental Immunization Activities (MSIAs) which took place in 2002 with a view for elimination of measles. The measles immunization coverage is considered to be medium $(50 \%-70 \%)$ and measles is responsible for a presumed low to medium mortality CFRO 0.5\% - 4\%). Also in place is the strategy for eradication of poliomyelitis by the year 2005 WHO, 1999b). The countries of the East African block recommended that to meet their strategies and achieve their goals in the control, eradication and elimination of the immunizable the following measures should be put in place (a) High routine immunization coverage of above 10\% (b) Conduct supplemental immunization activities (SIAs) with above $80 \%$ coverage for each round, (c) conduct supplemental National Immunization Days (NIDS) in selected high risk districts using appropriate strategy while strengthening case management, and an effective system for integrated disease surveillance and response for prompt and timely epidemic management[8].

With the launch of KEPI in 1980, for the acceleration of routine immunization in Kenya, tremendous progress has been achieved in the protection of children against the immunizable diseases. At its initiation, KEPI noted that immunization coverage in Kenya was at 30\% and by 1993 it had raised to 80\% [7]. This achievement was not maintained and in 1998, it had raised to $80 \%$ [7]. This achievement was not maintained and in 1998, KDHS showed a decline in the routine immunization coverage from 80\% in 1993 to $65 \%$ in 1998. This decline has caused an increase in immunizable or vaccine preventable diseases affecting children below 5 
years (ROK, 2001). Nyeri County reported areas with low immunization coverage notably in Mukurweini Division [7].

Despite the National Immunization Days (NIDs) for measles campaign known as the MSIAs in 2002 where the coverage in Nyeri County was $82 \%$ there were suspected cases of measles reported in Mukurweini. Blood samples from the cases were drawn and the specimens taken to KEMRI for laboratory analysis and results were received as measles Igm positive[8]. Despite availability of Global Alliance for Vaccine Initiative (GAVI) funds which have strengthened and enabled improvement in immunization in many areas, the routine immunization coverage for the under-fives is still below $80 \%$. This research therefore addressed the reasons responsible for low routine immunization coverage, the associated morbidity and mortality trends among children, the social economic, cultural and operational factors that impede strengthening of immunizations coverage in Nyeri County.

\subsection{The Study Area}

\section{Materials And Methods}

This study was carried out in Nyeri County in Mukurweini and Tetu Divisions.

\subsection{Study Population}

The study population consisted of mothers or caretakers, opinion leaders, District Health Management Team members and health workers in the immunizing health facilities.

\subsection{Inclusion Criteria}

Those included in the study were all children up to five years of age, birth cohorts who appeared for immunization at the time of the study, mothers/caretakers who with their children appeared at the immunizing health facilities, and all health workers who worked at the immunizing health facilities who consented to participate in the study.

\subsection{Exclusion Criteria}

Those excluded in the study were all children above 5 years of age at the time of the study, mothers/caretakers who with or without their children did not appear at the immunizing health facilities, all health workers who did not work at the immunizing health facilities other than the DHMT, and any respondent or interviewee who declined to participate in the study.

\subsection{Study Design}

The study was descriptive, cross section and collected both quantitative and qualitative data.

\subsection{Sample Size Determination and Sampling Techniques}

In the two divisions, all the records of routine immunization coverage for the children under five years were received against the data of the expected birth cohorts in the immunization clinics. This included those children immunized routinely. Selection of the study subjects for key informant interviews was done through purposive sampling technique while mothers or caretakers were selected form the clinics using systematic sampling. The sample size calculated using Firshers el al fomula was 400 respondents.

\subsection{Ethical considerations}

Clearance was sought from the following institutions: Kenyatta University and Ministry of Education, Science and Technology. Informed consent was obtained from the respondents with assurance of confidentially. Coded numbers were used instead of names of the subjects.

\subsection{Data Collection Methods and Research Instruments}

Before this study was carried out, research assistants were trained and pre-testing of research tools done in Nyeri town in a similar population. The actual collection of data was done over a period of three months.

\subsection{Focus Group Discussion}

Eight FGDs with mothers/ caretakers, opinion leaders, motivators (CBOs) and health workers in the health facilities were held; four in each division to give clarity on the factors captured in a one to one in depth interview.

\subsection{Data Management and Analysis}

Data collected from both divisions was coded before entry and captured using integrated microcomputer processing system (IMPS) version 3.1. This was done because the IMPS had fewer outliers and data editing is done on the screen during entry. Then the data was converted to SPSS Program version 15 through a written program. Chi- square test was used to determine associations between variables at $\mathrm{p}=0.05$ level of significance. The odds ratio estimates of $95 \%$ confidence interval for every model were used for comparison of strength of relationship between the variables as in multivariate regression. Qualitative data from each FGD was transcribed, coded and analyzed descriptively 


\section{Results}

A total of 412 respondents were interviewed on socio-cultural, economic and operational factors that influenced routine immunization in Nyeri County. 398 of these were mother/caretakers, eight were health workers and six were opinion leaders.

\subsection{Occupations of mothers/caregivers.}

Results of the socio-economic characteristics of the mothers/caregivers accompanying their children for immunization showed that $163(40.9 \%)$ were housewives, $112(28.1 \%)$ farmers, $52(13.2 \%)$ business women, $27(6.9 \%)$ students and 44(10.9\%) were involved in other economic activities (fig 4.5). There was a significant relationship between occupation of mothers and practice of immunization at $0.01 \%$ level, (x2 2.451, p,0.01, df 4)

Table 1: Occupation of mother/caregivers

\begin{tabular}{|l|l|l|l|l|l|l|}
\hline & Housewives & Farmers & Business & Any other & Student & Total \\
\hline Mukurweini & 88 & 48 & 20 & 26 & 16 & $\mathbf{9 8}$ \\
\hline Tetu & 75 & 64 & 32 & 18 & 11 & $\mathbf{2 0 0}$ \\
\hline Total & $\mathbf{1 6 3}$ & $\mathbf{1 1 2}$ & $\mathbf{5 2}$ & $\mathbf{4 4}$ & $\mathbf{2 7}$ & $\mathbf{3 9 8}$ \\
\hline
\end{tabular}

It was noted that the occupation of the mother/caretakers influenced the amount of money spent on transport to the immunization center. In Tetu, 10(2.5\%) respondents paid ten shillings, 22(5.6\%) paid twenty shillings, $13(3.3 \%)$ paid thirty shillings and 150(38\%) spent no money to reach the health facility. In Mukurweini, the amount of money spent on transport was lower with 3(0.76\%) mothers/caretakers spending ten shillings, $9(2.3 \%)$ paid twenty shillings, $6(1.5 \%)$ spent thirty shillings and $182(46.1 \%)$ spent no money to reach the healthy facility. There was no significant difference in amount of money used to reach to reach health facilities in the two divisions (x2 6.224, p, 0.05, df 3).

\subsection{Reasons hindering uptake of immunization as reported by mothers/caretakers.}

Eight hundred twenty four responses were received from the mothers/caretakers to establish the factors that influenced participation in routine immunization. Ninety nine (12\%) of mothers/caretakers in Tetu experienced long waiting time at the clinics as the major problem preventing them from presenting their children for immunization. In Mukurweini, 114(13.8\%) respondents had fear of side effects of immunization as their major hindrance.

Table 2. Factors influencing immunization as reported by mothers/caretakers

\begin{tabular}{|l|l|l|}
\hline Factors & Tetu(\%) & Mukurweni(\%) \\
\hline Culture not allow & 0.5 & 0.1 \\
\hline Busy with other work & 10.3 & 7.7 \\
\hline Lack of knowledge on immunization & 0.6 & 0.2 \\
\hline Religion not allow & 1.0 & 1.5 \\
\hline Child not taken for immunization & 4.0 & 9.3 \\
\hline Day not immunization not convenient & 0.9 & 0 \\
\hline Fear of side effects & 7.0 & 13.8 \\
\hline No faith in immunization & 0.7 & 0.7 \\
\hline Long waiting time & 12.0 & 6.4 \\
\hline Rumors about vaccine & 7.9 & 9.8 \\
\hline No response & 1.9 & 3.5 \\
\hline
\end{tabular}

\subsection{Health system factors influencing routine immunization coverage as reported by all respondents}

One hundred twenty six $(44.33 \%)$ of the mothers/caretakers in Tetu division and $136(55.67 \%)$ in Mukurweini indicated the lack of staff at the health facilities as a hindrance to routine immunizations. In the two divisions, a total of 262 out of $460(57 \%)$ responses from the mothers/caretakers singled out staff problem as their operational hindrance to children's immunization. Fifty two $(11.3 \%)$ of the respondents from Tetu and $73(15.9 \%)$ of the respondents from Mukurweini were not happy with the attitude of the health workers.

Table3. Health system factors influencing routine immunization coverage as reported by mothers/caretakers

\begin{tabular}{|l|l|l|}
\hline Response & Tetu & Mukurweini \\
\hline Attitude of health workers & 11.3 & 15.9 \\
\hline Lack of vaccine & 27.4 & 29.6 \\
\hline Lack of vaccine & 1.3 & 0 \\
\hline Not reminded by Health workers on dates & 2.4 & 0.2 \\
\hline No response & 4.6 & 7.4 \\
\hline
\end{tabular}




\subsection{Socio-economic factors influencing routine immunization.}

The socio-economic factors influencing immunization as indicated in focus group discussions (FGDs) by opinion leaders were long distances covered by the mothers/caretakers in Nyeri County to get to immunization centers and lack of money. The mothers/caretakers indicated lack of money (46.2\%), long distances to immunization centers $(40.5 \%)$ and the need pay for services.

Table 4. Mothers/caretakers socio-economic responses for barriers in routine immunization services.

\begin{tabular}{|l|l|l|}
\hline Problems & Mothers/caretakers & $\%$ \\
\hline Paying for services offered & 14 & 3.5 \\
\hline Long distance to immunization & 161 & 40.5 \\
\hline Lack of money & 184 & 46.2 \\
\hline No response & 77 & 19.3 \\
\hline
\end{tabular}

3.5 Factors influencing routine immunization as reported by health workers.

The eight workers interviewed were in the ages 20-24 years (12.5\%), 30-34 years (25\%), 40-44 years $(50 \%), 49$ and above (37.5\%). Two male and female health workers from each division were chosen. For this group of respondents, the socio-cultural factors influencing immunization in Nyeri County were rumors about the vaccine (100\%), long waiting time at the clinics $(50 \%)$, health condition of child (sick hence not taken for immunization) (37.5\%), fear of side effects (25\%), lack of knowledge of immunization (12.5\%), and no response $(25 \%)$.

The health workers indicated that the major factor that led to low routine immunization was unavailability of money and long distances and cost to the immunizing health facilities (100\%). At their respective health facilities, all the health workers $8(100 \%)$ reported lack of staff as a leading problem resulting into low routine immunizations. Three of the health workers (37.5\%), two from Mukurweini and one from Tetu division reported poor attitude of their fellow workers towards the mothers/caretakers as a contributing factor to low immunization coverage in Nyeri. The health workers gave various suggestions in order to improve mothers/caretakers attendance for routine immunizations (figure 4.10 and table 4.9)

\subsection{Interventions to increase immunization uptake}

Table 5. Corrective measures meant to increase immunization coverage as recommended by health workers.

\begin{tabular}{|l|l|l|}
\hline Opinion & Tetu $(\%)$ & Mukurweini(\%) \\
\hline Salary increment & 62.5 & 50 \\
\hline Employment of more staff & 50 & 37.5 \\
\hline Health education in barazas & 12.5 & 25 \\
\hline Mobile clinics & 12.5 & 37.5 \\
\hline Introduce mmr vaccine & 0 & 12.5 \\
\hline Staff transfers & 0 & 12.5 \\
\hline
\end{tabular}

Table 6. Corrective measures to improve immunization coverage as recommended by mothers/caretakers

\begin{tabular}{|l|l|l|}
\hline Corrective measures & Tetu (\%) & Mukurweini (\%) \\
\hline Salary increase for health workers & 12.6 & 8.5 \\
\hline Employ more staff & 14.0 & 10.6 \\
\hline Health education & 1.8 & 2.0 \\
\hline Build modern maternity wards & 10.1 & 0 \\
\hline Staff rotation and transfers & 0.3 & 7.9 \\
\hline Prompt payment to farmers produce & 0.1 & 0.7 \\
\hline Repair roads for communication & 10.3 & 9.8 \\
\hline
\end{tabular}

Some of the corrective measures to increase uptake of routine immunization as recommended by the health workers, mothers and caretakers included salary increment, employment of additional staff, mobile clinics and health education.

\section{Discussions}

\subsection{Age and sex related annual trends of routine immunization in Nyeri District}

The study showed that most $(88.7 \%)$ of the children immunized in both Mukurweini and Tetu division were aged $0-4$ months with Mukurweini having 9978 (50.5\%) while Tetu had 566 (38.2\%). The male to female ratio of the study population showed a 1:1.2 with a significant correlation of $0.01 \%$ level among health facilities. It was noted that whenever there was an increase in immunization of one sex, the same was reflected in the other sex. These results compare well with a study conducted in India, aimed at imparting surveillance, where there was a variation in sex of the cohorts under study[10]. 
Those children aged one month comprised $50 \%$ of the total children immunized. This immunization coverage could be due to vaccinations offered at both of the cohorts in those health facilities which have maternity wards. This makes it easier to capture all the children born at the maternities for immunization.

\subsection{Socio- Cultural Factors influencing routine immunizations}

There were several socio cultural factors that were identified as barriers to routine immunizations. The factors identified were affordability, accessibility and acceptability of the immunization services provided to the under five year old children. Of the two division, Mukurweini had the majority $438(53.2 \%)$ of the responses while Tetu had $386(46.8)$. $55(13.8 \%)$ of the respondents were males while $343(86.18 \%)$ were females.

The respondents who were mothers/caretakers could pick on one or more responses on the social cultural problems which prevented children from being presented to the Health facilities for immunization. Only $7(0.02 \%)$ of the respondents who included males $(13.82 \%)$ were outside the age bracket of mothers of childbearing age $(15-49$ yers $)$. though routine immunization services were free, there were those who could not access them because of long distance from there were those who could not access them because of long distance from their homes and poor roads. Similar findings were also reported in Siaya [11].

The majority of the respondents 172 (20.9\%) indicated that fear of side effects associated with vaccines was a major hindrance to immunization with Mukurweini having $14(66.3 \%)$ of the responses. This ALSo featured in the plan of action for Integrated Disease Surveillance and Response [12], which reports that disease outbreaks mostly affect those who avoid immunizations leading to high morbidity and mortality. Other respondents cited long waiting time while queing to get services as a major problem $(18.4 \%)$ as is the case in Tetu 99 (65.5\%) compared to Mukurweini 53 (34.5\%). A good number of responses 148 (18\%) noted being busy with other works could jeopardize chances of making children appear to the CWC for immunization. Some rumors which were associated with vaccines 146 (17.7\%) resulted in mothers /caretakers fear to get their children immunized. Most of the fear $(55.5 \%)$ was being felt in Mukurweini and the attached information was that the vaccines were mixed with drugs that would make children impotent when they grew up. This was said to be a form of family planning to reduce the population of people of Central Province since they were viewed as opponents of the government or antigovernment before the current government came to power. This affects disease surveillance and response activities as supported by the guideline for epidemic response on measles (WHO, 1999). It is worthy to note that since Nyeri District is an area of different religions, 20 (24\%) said their sects do not allow them to take their children for immunization or medical treatment. Out of these $12(60 \%)$ of the responses were from Mukurweini. A total of $110(13.3 \%)$ responses noted that, if a child is sick he/she should not be taken for immunizations. Seven $(0.8 \%)$ of the responses with $5(71.4 \%)$ of them from Tetu division indicated that they had never heard of existence of immunization. This was unlikely to be so since all the 7 respondents who mav be the responses were in the different Clinics for immunizations. Another $7(0.8 \%)$ of the responses which were all from Tetu indicated that immunization days were inconvenient with their calendar of events. These results compare well with the findings of a study on factors influencing measles immunization coverage in Siaya District (Odanga, 2003) where mothers /caretakers were only available on market days for immunization. Only $12(1.5 \%)$ with 50\% responses from each division aired their views in not having faith in immunizations. They preferred herbal care for any disease than the conventional treatment or immunizations. In all the responses, $45(11.3 \%)$ respondents out of 398 did not tick for any response. Only 5 $(0.6 \%)$ with majority $4(80 \%)$ from Tetu said culture does not allow them to take their children for immunization.

\subsection{Social economic determinants of routine immunization.}

Those interviewed gave different views on factors that determine routine immunizations. In the facilities, the amount of money paid for the services and lack of money were a barrier in accessing routine immunization services for the under five year old children. The majority of mothers /caretakers $(75 \%)$ walked to the immunizing health facilities.

These are the ones who found it easier to walk to the health facilities since they could not afford to pay for any means of transport such as matatus, bicycles or private vehicles. Only $58(14.6 \%)$ of the respondents used matatus. Due to the cold weather and poor roads which are muddy and chilly, not many respondents used bicycles for transport. So only 2 (28.6\%) mothers /caretakers used bicycles in Mukurweini and 7 (71.4\%) use them in Tetu. At least $32(8 \%)$ of the respondents used vehicles to bring thir children for immunization than their counterparts in Mukurweini. These findings explain that the transport system is more available, accessible, and affordable in Tetu compard to Mukurweini Division.

\subsection{Health systems determinants of Routine Immunization}

There were some major determinants identified as setbacks to high coverage of routine immunization in the operational areas. Some respondents gave more than one response. A total of 402 responses were received 
from the mothers /caretakers, with $55(13.8 \%)$ respondents' abstentions. Of those received, 192 (47.8\%) responses were from Tetu while 210 (52.2\%) were from Mukurweini.

Poor and unethical attitude of health workers was cited as a hindrance to routine immunization by 125 (31.1\%) responses with Tetu giving $52(41.6 \%)$ and Mukurweini 73 (58.4\%). This attitude of health workers which was said to be unacceptable could be due to cover work and understaffing in the health facilities. That is why $262(65.2 \%)$ of the responses cited lack of staff as a major contributory factor to the barrier in accessing routine immunizations whose effect is almost equally felt in the two divisions. There were only $6(1.5 \%)$ responses which indicted lack of vaccines as a barrier to routine immunizations, all (100\%) of who were from Tetu and none $(0 \%)$ from Mukurweini. There was a time when B.C.G. vaccine was out of stock in the vaccine stores in Nyeri. Lack of supply of drugs and vaccines in time may have contributed to the low immunization coverage. This had earlier been mentioned by the department of vaccines and biological (WHO, 1999 B), that the untimely supply of drugs and vaccines or lack of their supply contributed to low immunization coverage. This crisis in the lack of BCG vaccines was also said to have been reported in other areas of the country. Though short lived, many children who would have been immunized were not done so and at the time required leading to low routine coverage. Twelve (3\%) of the responses cited they were not reminded of the return data for the next vaccination by the health workers. Of these, 11(91.7\%) responses were form Tetu and $1(8.3 \%)$ from Mukurweini. This could be attributed to the much work in the coffee and tea farms which would make the respondents forget the date of next appointment which is normally written in their clinic cards.

\subsection{Interventions measures proposed for strengthening routine immunization}

A total of 895 responses were received from the mothers /caretakers citing various proposed measures. Ninety five (42.8\%) of the responses were from Mukurweini and 127 (57.2\%) were from Tetu. This related well with corrective measures cited form Tetu and corrective measures cited by opinion leaders 4 (26.7\%). Salary increase to health workers was proposed by $189(21.1 \%)$ responses as an important measure in strengthening routine immunization. This had also been reported in a report on review of EPI African regional workshop on strengthening immunization [3] which stated that the salary increases for health workers can be a very important intervention because of the enormous amount of work experienced by the few available health workers. The staff shortages were evident in all health facilities during the study period. These results suggest that there is need to review employment strategies and salaries for health workers.

Repairs of the rural access roads was proposed by $180(20.1 \%)$ for easy accessibility to the health facilities. The majority of the mothers /caretakers (75\%) walk on foot, but with the roads repair many vehicles will traverse the interior to pick on the passengers. All the 90 (100\%) responses who proposed building, modernizing and equipping maternity wards in all health facilities came from Tetu. The respondents in the category may have had in mind that to achieve a good immunization coverage, newborns must be targeted at birth for vaccinations; seventy (7.8\%) responses indicated that starting mobile clinics in the district would be strengthening measure in achieving high routine immunization status. This is also supported by a book on introduction to IMCI [12] which cites mobile clinics as a way of increasing immunization coverage. A total of $28(3.1 \%)$ respondents proposed that doctors /clinicians should be posted to all health facilities. They mentioned that they are needed more so for treatment of pediatric illnesses and as a measure of attracting more children on the health facilities C.W.C.s. Health education and community sensitization in Barazas was mentioned as an intervention measure by $34(3.8 \%)$ responders. The health education may take place as a continuous micro teaching exercise in the MCH/FP Clinic where CWC services take place. The two of them should take place on market days also. This compares well with information in child health book (AMREF, 1993) which emphasizes on health education to mothers in MCH/FP clinics. This will ensure creation of awareness especially to the upcoming mothers as was the case with the $76(0.8 \%)$ mothers /caretakers who had never heard of existence of immunization.

About $74(8.3 \%)$ responses cited staff rotations, changes and transfers as a measure to rid of the unproductive health workers so as to improve immunization services. Of these, $71(96 \%)$ responses were from Mukurweini. Mukurweini could be having un cooperative rude or reluctant health workers prompting the mentioned corrective measures Eight $(0.9 \%)$ of the responses suggested the need for better payment for their farm produce for them to get money to enable them get good transport and for other dividends. This was so especially in Mukurweini division where $7(87 \% .5 \%)$ of the responses came from. This could be so due to the existing poor living standards in the division. This supported by a special report on immunization in Kenya (KDHS, 2003), which showed that poverty contributed a lot to low immunization.

Health workers were in support of the corrective measures indicated by the mothers/caretakers, though salary increment came first $(36 \%)$ in the order of their proposals. The employment of more staff was also cited as an alleviating measure to improve immunization with $7(28 \%)$ responses. Other measures were Health Education in Barazas (12\%) re-establishment of mobile clinic services (16\%) while both introductions of MMR vaccine and staff transfers had $4 \%$ responses for each. The opinion leaders supported the proposals of the 
mothers, caretakers and the Health Workers. About $25 \%$ of the responses highly sported employment of more health workers while $12.5 \%$ viewed increase of salary for health workers as a measure to increase immunization coverage. Three $(18.7 \%)$ of the responses cited establishment of mobile clinics as an alleviating measure, so as to reach people with difficulties of travel. These responses came from Tetu and none from Mukurwini. Some responses $(12.5 \%)$ from opinion leaders especially those of Mukurweini division suggested free medical services to mothers, as a measure of attracting the unimmunized children to accompany their mothers /caretakers to the MCH/FP clinic and C.W.C. This would increase chance of children being immunized at the C.W.C. other responses targeted staff retention (6.25\%) and posting of a doctor or clinician (6.25\%) to all the health facilities. Building of a functional maternity ward in every health facility was viewed as a measure to boost immunization of the newborn children by the opinion leaders' responses $(12.5 \%)$ offering free medical services was more likely to improve routine immunization coverage than intensifying mobile outreach clinics as mentioned by many opinion leaders. This compares well with a study on morbidity and mortality in Sub Saharan Africa Feacham and Jamison, [13] which showed that immunization reduces diseases and deaths form immunizable diseases.

\section{Conclusion and recommendations}

The main factors that influenced routine immunizations in the two divisions of Mukurweini and Tetu were economic, social, cultural and operational. With effective corrective measures and cooperation between the stakeholders and health services providers, strengthening of the routine immunizations services was mentioned by responders to be achievable task. There is need for networking between Community Based Organization (CBOs), Provincial Administration, NGOs and other health sector actors for strengthening intersectoral collaboration to enhance effective implementation of programs to sensitize the public on importance of routine immunization.

\section{References}

[1]. World Health Organization (1999b). Department of Vaccines and Biologicals Consensus Meeting on Assessment and Monitoring of Vaccine Preventable Diseases. WHO/

[2]. Pan American Health Organization (1999). Measles Eradication Field Guide. PAHO/B/99.41:1 - 100.

[3]. Anderson J.R. (1985). Muirs Textbook of Pathology. 12th Edition Pg. 62.

[4]. Barbara M., Tony L., Ross J and Ian R. (2001). Changes in Expanded Programme for Immunization Coverage for Mother and Child in Krakor, Cambodia 1996 - 1998, 6 (7): 526.

[5]. African Medical Research Foundations (1993). Child Health Chapter 13 Pg. 23.

[6]. Burstrum B., Aaby P., and Nytue (1995). A Review of Studies on Incidence, Vaccine Efficacy and Mortality in East Africa. E.A. Medical Journal 72 (3) 155.

[7]. Central Bureau of Statistics (1999). Population And Housing Census volume 1. Ministry of Finance and Planning Page 5 - 30.

[8]. Cook G.G. (1996). Manson's Tropical Diseases 20th Edition. Pages 244: 787. Publisher.

[9]. Kenya Expanded Programme on Immunization (2003). Performance Monitoring Handbook Chapter 6 PG. 26

[10]. Thankur J. (2002). Factors influencing the Imparting of Surveillance ifn India

[11]. Odanga J. (2003). Factors influencing measles immunization in Siaya Pg. $1-50$.

[12]. Republic of Kenya - MOH (2003). Integrated Management of Childhood Illnesses. Introduction MOH/WHO/UNICEF/IMCI/:1

[13]. Kenya Demographic Health Survey (2003). Health Survey: Special Report on Immunization. 